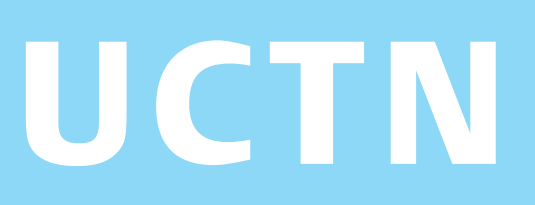

\title{
Retroperitoneal perforation caused by endoscopic biopsy in a patient with ulcerative colitis and cyto- megalovirus infection
}

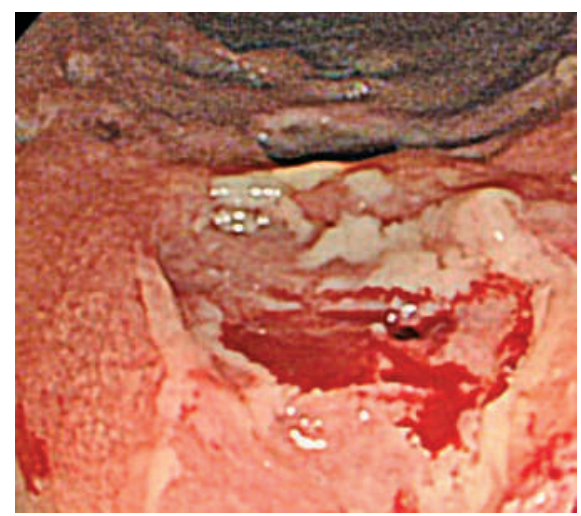

Figure 1 An endoscopic view showing the pinhole perforation of the base of the rectal ulcer.

A 52-year-old woman was admitted to our hospital for initiation of treatment for ulcerative colitis. She was given 2week course of ganciclovir because she was positive for cytomegalovirus (CMV) antigens. Colonoscopy was performed 2 months after she started prednisolone treatment, which showed multiple ulcers with a well-defined, punched-out appearance, erosions, and diffuse redness in the colon. The colonoscopic findings were consistent with ulcerative colitis exacerbated by CMV infection. Multiple biopsy specimens were taken using a standard biopsy forceps (2.2-mm cup, non-spiked). A pinhole-like perforation occurred at the time of the biopsy of the rectal ulcer base (Figure 1). Computed tomography revealed a pneumoretroperitoneum (Figure 2). A diagnosis of retroperitoneal perforation was made and she was treated with an antibiotic and remained on a nilby-mouth regime for 1 week. Because the patient still tested positive for CMV antigens, a 2-week course of treatment with gancyclovir was started on day 71 . The patient made a rapid and uneventful recovery and was discharged on day 84 .

The biopsy specimen of the perforation site was small in size and contained only ulcer-bed tissue without submucosa or muscularis propria. Biopsy-related perforations are rare complications of intestinal endoscopy. Foliente et al. [1] reported three cases of cecal perforation caused

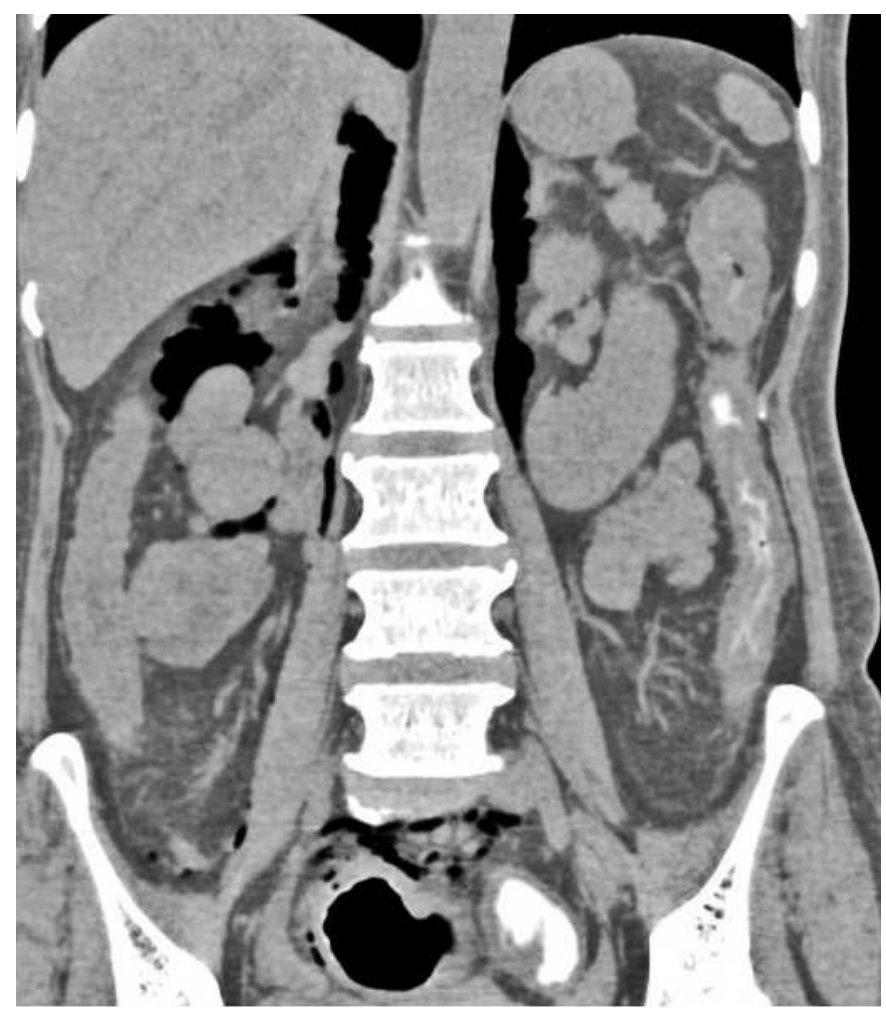

Figure 2 A computed tomographic scan showed a pneumoretroperitoneum. by routine biopsy of inflammatory or ulcerative lesions. Volker et al. [2] reported a case of colonic perforation in a patient with ulcerative colitis where biopsy specimens contained muscularis propria with severe mucosal atrophy.

Many instances of perforation in cases of CMV-related ulcer, some of them in patients with ulcerative colitis, have been reported and these ulcers are suspected to have been caused by vascular ischemia of the gut wall $[3,4]$. The colorectal wall may be extremely thin at the ulcer base and may be liable to rupture. We should therefore perform endoscopic biopsy in such patients with extreme care or should not biopsy at all if CMV infection is obvious on the basis of endoscopic findings or CMV antigenemia.

Endoscopy_UCTN_Code_CPL_1AJ_2AB

\section{H. Miyatani, Y. Yoshida}

Department of Gastroenterology, Jichi Medical University, Omiya Medical Center, Omiya, Saitama, Japan.
References

${ }^{1}$ Foliente RL, Chang AC, Youssef AI et al. Endoscopic cecal perforation: mechanisms of injury. Am J Gastroenterol 1996; 91: 705-708

2 Eckardt VF, Gaedertz C, Eidner C. Colonic perforation with endoscopic biopsy. Gastrointest Endosc 1997; 46: 560-562

${ }^{3}$ Iwasaki T. Alimentary tract lesion in cytomegalovirus infection. Acta Pathol Jpn 1987; 37 : 549-565

${ }^{4}$ Kishore J, Ghoshal U, Ghoshal UC et al. Infection with cytomegalovirus in patients with inflammatory bowel disease: prevalence, clinical significance and outcome. J Med Microbiol 2004; 53: 1155 - 1160

\section{Corresponding author}

\section{H. Miyatani, MD}

Department of Gastroenterology

Jichi Medical University · Omiya Medical Center · 1-847 Amanuma · Omiya · Saitama 330-8503 · Japan

Fax: $\quad+81-48-648-5188$

Email: miyatani@omiya.jichi.ac.jp

DOI: $10.1055 / s-2007-967010$ 